\title{
Quasisymmetrically inequivalent hyperbolic Julia sets
}

\author{
Peter Haïssinsky and Kevin M. Pilgrim
}

\begin{abstract}
We give explicit examples of pairs of Julia sets of hyperbolic rational maps which are homeomorphic but not quasisymmetrically homeomorphic.
\end{abstract}

\section{Introduction}

Quasiconformal geometry is concerned with properties of metric spaces that are preserved under quasisymmetric homeomorphisms. Recall that a homeomorphism $h: X \rightarrow Y$ between metric spaces is quasisymmetric if there exists a distortion control function $\eta:[0, \infty) \rightarrow[0, \infty)$ which is a homeomorphism and which satisfies $|h(x)-h(a)| /|h(x)-h(b)| \leq \eta(|x-a| /|x-b|)$ for every triple of distinct points $x, a, b \in X$. We shall say that $X$ and $Y$ are quasisymmetrically equivalent if there exists such a homeomorphism.

A basic - even if still widely open - question is to determine whether two given spaces belong to the same quasisymmetry class, once it is known that they are homeomorphic and share the same qualitative geometric properties. This question arises also in the classification of hyperbolic spaces and word hyperbolic groups in the sense of Gromov [5], [14], [10]. Besides spaces modelled on manifolds, very few examples are understood; see nonetheless [4] for examples of inequivalent spaces modelled on the universal Menger curve. Here, we focus our attention on compact metric spaces that arise as Julia sets of rational maps. A rational map is hyperbolic if the closure of the set of forward orbits of all its critical points does not meet its Julia set. We address the question of whether the geometry of the Julia set of a hyperbolic rational map is determined by its topology. More precisely, given two hyperbolic rational maps $f$ and $g$ with homeomorphic Julia sets $J_{f}$ and $J_{g}$, does there exist a quasisymmetric homeomorphism $h: J_{f} \rightarrow J_{g}$ ?

Hyperbolic Julia sets serve our purposes for several reasons. First, it rules out elementary local obstructions. For instance, the Julia set of $f(z)=z^{2}$ is the Euclidean unit circle $\mathbb{S}^{1}$, while that of $g(z)=z^{2}+1 / 4$ is a Jordan curve with a cusp 
at the unique fixed point, so they are not quasisymmetrically equivalent. Second, if $f$ is hyperbolic, it is locally invertible near $J_{f}$, and the inverse branches are uniformly contracting; the Koebe distortion theorem then implies that $J_{f}$ satisfies a strong quasi-self-similarity property. Among such maps, in some cases, this implies that homeomorphic Julia sets are quasisymmetrically homeomorphic.

1. If the Julia set of a hyperbolic rational map is a Jordan curve, then it is quasisymmetrically equivalent to the unit circle [23].

2. Let $C \subset \mathbb{R}$ denote the usual middle thirds Cantor set. Recall that any compact, totally disconnected metric space without isolated points is homeomorphic to $C$; see e.g. Theorem 2.97 in [13]. If the Julia set of a hyperbolic rational map is homeomorphic to $C$, then, by a theorem of David and Semmes (Proposition 15.11 in [8]) they are quasisymmetrically equivalent.

3. If $f$ and $g$ are hyperbolic and their Julia sets are homeomorphic by the restriction of a global conjugacy, then they are also quasisymmetrically equivalent by Theorem 2.9 in [19].

So one must look to more complicated Julia sets for potential examples of quasisymmetrically inequivalent Julia sets.

We will show:

Theorem 1. Let $f(z)=z^{2}+10^{-9} / z^{3}$ and $g(z)=z^{2}+10^{-20} / z^{4}$. Then $J_{f}, J_{g}$ are each homeomorphic to $C \times S^{1}$, but they are not quasisymmetrically homeomorphic.

Recall that a metric space $X$ equipped with a Radon measure $\mu$ is Ahlfors regular of dimension $Q$ if the measure of a ball satisfies $\mu(B(x, r)) \asymp r^{Q}$; one has then that $X$ has locally finite Hausdorff measure in its Hausdorff dimension, $Q$. Its conformal dimension $\operatorname{confdim}(X)$ is the infimum of the Hausdorff dimensions of all metric spaces quasisymmetrically equivalent to $X$. As an invariant of the quasisymmetry class of a metric space, conformal dimension and other variants such as the Ahlfors-regular conformal dimension have been the subject of much recent investigation; see e.g. [15]. Since the Julia set of any hyperbolic rational map is quasi-self-similar, it follows that it is Ahlfors regular and porous, hence has Hausdorff dimension strictly less than 2 by Theorem 4 and its corollary in [24]. So if $f$ is hyperbolic then $\operatorname{confdim}\left(J_{f}\right)<2$. We prove Theorem 1 by showing $\operatorname{confdim}\left(J_{f}\right) \neq \operatorname{confdim}\left(J_{g}\right)$.

The arguments we use to prove Theorem 1 will generalize to yield:

Theorem 2. There exist hyperbolic rational maps each of whose Julia sets is homeomorphic to $C \times S^{1}$ and whose conformal dimensions are arbitrarily close to 2 .

It follows that there exists an infinite sequence of hyperbolic rational maps whose Julia sets are homeomorphic to $C \times S^{1}$ but which are pairwise quasisymmetrically inequivalent.

Our method of proof of Theorem 2 requires that our examples be rational functions whose degrees become arbitrarily large. It is tempting to look for a sequence of simpler examples. Polynomials will not work: as is shown by Carrasco [7], the 
conformal dimension of any hyperbolic polynomial with connected Julia set is equal to 1 .

If connected, the Julia sets of hyperbolic polynomials have many cut points. At the opposite extreme, recall that a Sierpiński carpet may be defined as a topologically one-dimensional, connected, locally connected compact subset of the sphere such that the components of its complement are Jordan domains with pairwise disjoint closures; any two such spaces are homeomorphic [25]. Sierpiński carpets are one-dimensional analogs of Cantor sets. They also play an important role in complex dynamics and hyperbolic geometry [17], [1]. Sierpiński carpets which arise from hyperbolic groups and hyperbolic rational maps also share the same qualitative properties: their peripheral circles are uniform quasicircles and are uniformly separated; Bonk also proved that any such carpet is quasisymmetrically equivalent to one where the complementary domains are round disks in $\widehat{\mathbb{C}}[2]$. Nonetheless, using similar methods, we will show:

Theorem 3. There exist hyperbolic rational maps with Sierpiński carpet Julia sets whose conformal dimensions are arbitrarily close to 2.

To our knowledge, an analogous result for conformal dimensions of limit sets of convex cocompact Kleinian groups is not yet known.

On the one hand, it is perhaps not surprising that there is a plethora of quasisymmetrically distinct Julia sets: any quasisymmetric map between round convex cocompact Kleinian group carpets is the restriction of a Möbius transformation according to Theorem 1.1 in [3]. Also, Theorem 8.1 in [1] asserts that any quasisymmetric automorphism of the standard square "middle ninths" carpet is the restriction of a Euclidean isometry. On the other hand, the proofs of these results are rather involved.

The proofs of our results rely on the computation of the conformal dimension of certain metric spaces homeomorphic to $C \times S^{1}$, following the seminal work of Pansu [21]. We will also make frequent use of the fact that on the Euclidean 2-sphere, an orientation-preserving self-homeomorphism is quasiconformal if and only if it is quasisymmetric; see Theorem 11.14 in [12]. We denote by $\mathbb{S}^{2}$ the round Euclidean 2-sphere.

The special case needed for the present purpose is summarized in $\S 1$. The proofs of the theorems appear in $\S \S 2$ and 3 .

Acknowledgement. We are grateful for the comments of the anonymous referee.

\section{Annulus maps}

Let $I=[0,1]$ and let $\iota: I \rightarrow I$ be the involution given by $\iota(x)=1-x$. Identify $\mathbb{S}^{1}$ with $\mathbb{R} / \mathbb{Z}$. We give $I \times \mathbb{S}^{1}$ the product orientation. Fix an even integer $m \geq 2$ and let $\mathcal{D}:=\left(d_{0}, \ldots, d_{m-1}\right)$ be a sequence of positive integers such that $\sum_{i=0}^{m-1} \frac{1}{d_{i}}$ $<1$. Then there exist real numbers $a_{i}, b_{i}, i=0, \ldots, m-1$ such that for each $i$, $\left|b_{i}-a_{i}\right|=\frac{1}{d_{i}}$ and

$$
0<a_{0}<b_{0}<a_{1}<b_{1}<\cdots<a_{m-1}<b_{m-1}<1 .
$$


Fix such a choice $a_{0}, b_{0}, \ldots, a_{m-1}, b_{m-1}$. For each $i$, let $J_{i}=\left[a_{i}, b_{i}\right]$, and let $g_{i}: I \rightarrow J_{i}$ be the unique affine homeomorphism which is orientation-preserving if $i$ is even and is orientation-reversing if $i$ is odd. This iterated function system on the line has a unique attractor $C(\mathcal{D})$ and its Hausdorff dimension, by the pressure formula given by Theorem 5.3 in [9], is the unique real number $\lambda=\lambda(\mathcal{D})$ satisfying

$$
\sum_{i=0}^{m-1} \frac{1}{d_{i}^{\lambda}}=1 .
$$

Let

$$
\tilde{F}:\left(\sqcup_{i=0}^{m-1} J_{i}\right) \times \mathbb{S}^{1} \rightarrow I \times \mathbb{S}^{1}=: A .
$$

be the map whose restriction to the annulus $A_{i}:=J_{i} \times \mathbb{S}^{1}$ is given by

$$
\left.\tilde{F}\right|_{A_{i}}(x, t)=\left(g_{i}^{-1}(x),(-1)^{i} d_{i} \cdot t \bmod 1\right) .
$$

That is, $\left.\tilde{F}\right|_{A_{i}}$ is an orientation-preserving covering map of degree $d_{i}$ which is a Euclidean homothety with factor $d_{i}$ and which preserves or reverses the linear orientation on the interval factors according to whether $i$ is even or, respectively, is odd.

The invariant set associated to $\tilde{F}$ is

$$
X(\mathcal{D}):=C(\mathcal{D}) \times \mathbb{S}^{1}=\bigcap_{n \geq 0} \tilde{F}^{-n}(A) .
$$

From Section 3 of [11], we have

Proposition 1.1. The conformal dimension of $X(\mathcal{D})$ is equal to $1+\lambda(\mathcal{D})$.

This statement is a particular case of a well-known general fact: if $X$ is a $\lambda$ Ahlfors regular metric space, then $X \times[0,1]$ equipped with the product metric has conformal dimension $1+\lambda$. This criterion is originally due to Pansu, see Proposition 2.9 in [21]; see also Proposition 3.7 in [10] and Tyson's Theorem 15.10 in [12].

\section{Proofs of Theorems 1 and 2}

Let $\mathcal{D}$ be a sequence of positive integers defining a family of annulus maps $\tilde{F}$ as in the previous section, and put $X=X(\mathcal{D})$.

Proposition 2.1. There is a smooth embedding $A \hookrightarrow \mathbb{S}^{2}$ such that (upon identifying $A$ with its image) the map $\tilde{F}: \sqcup_{i} A_{i} \rightarrow A$ extends to a smooth map $F: \mathbb{S}^{2} \rightarrow \mathbb{S}^{2}$ whose iterates are uniformly quasiregular. There is a quasiconformal (equivalently, a quasisymmetric) homeomorphism $h: \mathbb{S}^{2} \rightarrow \widehat{\mathbb{C}}$ such that $h \circ F \circ h^{-1}$ is a hyperbolic rational map $f$, and $h(X)=J_{f}$, the Julia set of $f$.

Proof. The existence of the extension $F$ is a straightforward application of quasiconformal surgery. We merely sketch the ideas and refer to [22] for details; see also the forthcoming text [6] devoted to this topic. The next two paragraphs outline this construction.

The linear ordering on the interval $I$ gives rise to a linear ordering on the set of $2 m$ boundary components of the set of annuli $A_{0}, \ldots, A_{m-1}$. We may regard $A$ 
as a subset of a smooth metric sphere $S^{2}$ conformally equivalent to $\mathbb{S}^{2}$. For $i=$ $1, \ldots, m-1$ let $C_{i}$ be the annulus between $A_{i-1}$ and $A_{i}$. Let $D_{0}, D_{1}$ be the disks bounded by the least, respectively greatest, boundary of $A$, so that the interiors of $D_{0}, A, D_{1}$ are disjoint. Let $D_{0}^{\prime}$ be the disk bounded by the least component of $A_{0}$ and $D_{1}^{\prime}$ be the disk bounded by the greatest component of $A_{m-1}$.

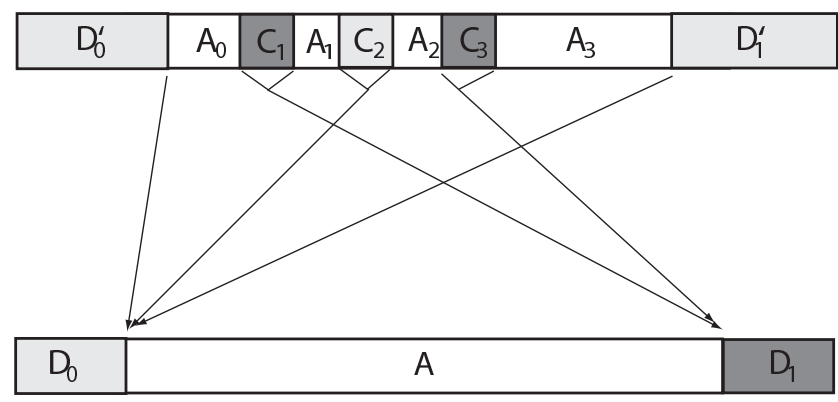

Figure 1. Caricature of the extended mapping, $F$.

We now extend $\tilde{F}$ as follows. See Figure 1 . Send $D_{0}^{\prime}$ to $D_{0}$ by a proper map of degree $d_{0}$ ramified over a single point $x$, so that in suitable holomorphic coordinates it is equivalent to $z \mapsto z^{d_{0}}$ acting near the origin; thus $D_{0} \subset D_{0}^{\prime}$ is mapped inside itself. Similarly, send $D_{1}^{\prime}$ to $D_{0}$ by a proper map of degree $d_{m-1}$ ramified only over $x$, so that in suitable holomorphic coordinates it is equivalent to $z \mapsto$ $1 / z^{d_{m-1}}$ acting near infinity; thus $D_{1} \subset D_{1}^{\prime}$ is mapped into $D_{0}$. To extend over the annulus $C_{i}$ between $A_{i-1}$ and $A_{i}$, note that both boundary components of $C_{i}$ map either to the least, or to the greatest, component of $\partial A$. It is easy to see that there is a smooth proper degree $d_{i-1}+d_{i}+1$ branched covering of $C_{i}$ to the corresponding disk $D_{0}$ (if $i$ is even) or $D_{1}$ (if $i$ is odd). This completes the definition of the extension $F$.

It is easy to arrange that $F$ is smooth, hence quasiregular. We may further arrange that the locus where $F$ is not conformal is contained in a small neighborhood of $C_{1} \cup \cdots \cup C_{m-1}$. This locus is nonrecurrent, so the iterates of $F$ are uniformly quasiregular. By a theorem of Sullivan (Theorem 9 in [24]), $F$ is conjugate via a quasiconformal homeomorphism $h: S^{2} \rightarrow \widehat{\mathbb{C}}$ to a rational map $f$. By construction, every point not in $h(X)$ converges under $f$ to a superattracting fixed point $h(x)$ in the disk $h\left(D_{0}\right)$, so $f$ is hyperbolic and $h(X)=J_{f}$.

We now establish a converse.

Proposition 2.2. Suppose $f: \widehat{\mathbb{C}} \rightarrow \widehat{\mathbb{C}}$ is a rational map for which there exist a closed annulus $A$ and essential pairwise disjoint subannuli $A_{0}, A_{1}, \ldots, A_{m-1}$, $m$ even, contained in the interior of $A$ such that (with respect to a linear ordering induced by $A$ ) $A_{0}<A_{1}<\cdots<A_{m-1}$. Let $D_{0}$, respectively $D_{1}$, be the disk bounded by the least, respectively greatest, boundary component of $A$. Further, suppose that for each $i=0, \ldots, m-1,\left.f\right|_{A_{i}}: A_{i} \rightarrow A$ is a proper covering map of degree $d_{i}$, 
with $f$ mapping the greatest component of $A_{i}$ and the least component of $A_{i+1}$ to the boundary of $D_{1}$ if $i$ is even, and to the boundary of $D_{0}$ if $i$ is odd. Put $\mathcal{D}=\left(d_{0}, d_{1}, \ldots, d_{m-1}\right)$. Let $\tilde{f}=\left.f\right|_{\bigsqcup_{i=0}^{m-1} A_{i}}$ and put $Y=\bigcap_{n \geq 0} \tilde{f}^{-n}(A)$. Then $Y \subset J_{f}, \tilde{f}(Y)=Y=\tilde{f}^{-1}(Y)$, and there is a quasisymmetric homeomorphism $h: Y \rightarrow X$ conjugating $\left.\tilde{f}\right|_{Y}: Y \rightarrow Y$ to $\left.\tilde{F}\right|_{X}: X \rightarrow X$ where $\tilde{F}$ is the family of annulus maps defined by the data $\mathcal{D}$.

Proof. The conformal dynamical systems of annulus maps defined by $\tilde{f}$ and by $\tilde{F}$ are combinatorially equivalent in the sense of McMullen (see Appendix A in [18]), so by Theorem A.1 in [18] there exists a quasiconformal (hence quasisymmetric) conjugacy $\tilde{h}$ from $\tilde{f}$ to $\tilde{F}$; we set $h=\left.\tilde{h}\right|_{Y}$.

Combined with Proposition 1.1, this yields:

Corollary 2.1. Under the assumptions of Proposition 2.2, $\operatorname{confdim}\left(J_{f}\right) \geq 1+$ $\lambda(\mathcal{D})$, with equality if $Y=J_{f}$.

Proof of Theorem 1. For $\epsilon \in \mathbb{C}$ let $f_{\epsilon}(z)=z^{2}+\epsilon / z^{3}$. In Section 7 of [16], McMullen shows that for $|\epsilon|$ sufficiently small the map $f_{\epsilon}$ restricts to a family of annulus maps with the combinatorics determined by the data $\mathcal{D}=(2,3)$ and with Julia set homeomorphic to the repellor $X_{(2,3)}$ determined by $\mathcal{D}$; it is easy to see that $\epsilon=10^{-9}$ will do.

Exactly the same arguments applied to the family $g_{\epsilon}(z)=z^{2}+\epsilon / z^{4}$ show that if $|\epsilon|$ is sufficiently small, the family $g_{\epsilon}$ restricts to a family of annulus maps with the combinatorics determined by $\mathcal{D}=(2,4)$ and whose Julia set is homeomorphic to the corresponding repellor $X_{2,4}$. It is easy to see that $\epsilon=10^{-20}$ will do; one may take $A=\left\{10^{-6}<|z|<10^{10}\right\}$. By Corollary 2.1 and Proposition 1.1, the conformal dimensions $1+\lambda_{f}, 1+\lambda_{g}$ of $J_{f}, J_{g}$ satisfy the respective equations $2^{-\lambda_{f}}+3^{-\lambda_{f}}=1$, $2^{-\lambda_{g}}+4^{-\lambda_{g}}=1$ and are therefore unequal. Since the conformal dimension is a quasisymmetry invariant, the proof is complete.

Proof of Theorem 2. For an even integer $n \geq 4$, let $\mathcal{D}_{n}=\left(d_{0}, d_{1}, \ldots, d_{n-1}\right)$ where $d_{0}=n+1$ and $d_{i}=n$ for $i=1, \ldots, n-1$. Let $f_{n}$ be the rational map given by Proposition 2.1. By Corollary $2.1 \operatorname{confdim}\left(J_{f_{n}}\right)$ is 1 plus the unique positive root $\lambda_{n}$ of the equation

$$
(n+1)^{-\lambda}+(n-1) n^{-\lambda}=1 .
$$

The left-hand side is larger than 1 when $\lambda=\frac{\log (n-1)}{\log (n)}$, so $\lambda_{n}>\frac{\log (n-1)}{\log n}$ and thus $\lambda_{n} \rightarrow 1$ as $n \rightarrow \infty$. Hence confdim $\left(J_{f_{n}}\right) \rightarrow 2$ as $n \rightarrow \infty$.

\section{Proof of Theorem 3}

Fix an even integer $n \geq 2$. For each such $n$, we will build a rational function $f_{n}: \widehat{\mathbb{C}} \rightarrow \widehat{\mathbb{C}}$ with the following properties: (1) its Julia set is homeomorphic to the Sierpiński carpet, and (2) there exist an annulus $A \subset \widehat{\mathbb{C}}$, and parallel pairwise disjoint essential subannuli $A_{0}, \ldots, A_{n-1}$ such that for each $i=0, \ldots, n-1$, the 
restriction $\left.f\right|_{A_{i}}: A_{i} \rightarrow A$ is a proper holomorphic covering of degree $(n+4)$, just as in the previous section. Theorem 3 will then follow immediately from Corollary 2.1 with $\mathcal{D}=(\underbrace{n+4, \ldots, n+4}_{n})$.

We will first define abstract Riemann surfaces $X$ and $Y$, an isomorphism $\varphi$ : $Y \rightarrow \widehat{\mathbb{C}}$, and an isomorphism $h: Y \rightarrow X$. The construction of the Riemann surface $X$ will depend on $n$. Next, we will define a holomorphic map $g: X \rightarrow Y$. The composition $F=g \circ h: Y \rightarrow Y$ will yield a dynamical system; $f_{n}=\varphi \circ F \circ \varphi^{-1}$ is the desired rational function. We are grateful to Daniel Meyer for suggesting this construction which is more explicit than our original one.

Below, it will be useful to identify the complex plane $\mathbb{C}$ with $\mathbb{R}^{2}$ in the usual way: $x+i y \leftrightarrow(x, y)$. For $z \in \widehat{\mathbb{C}}$ set $j(z)=\bar{z}$.

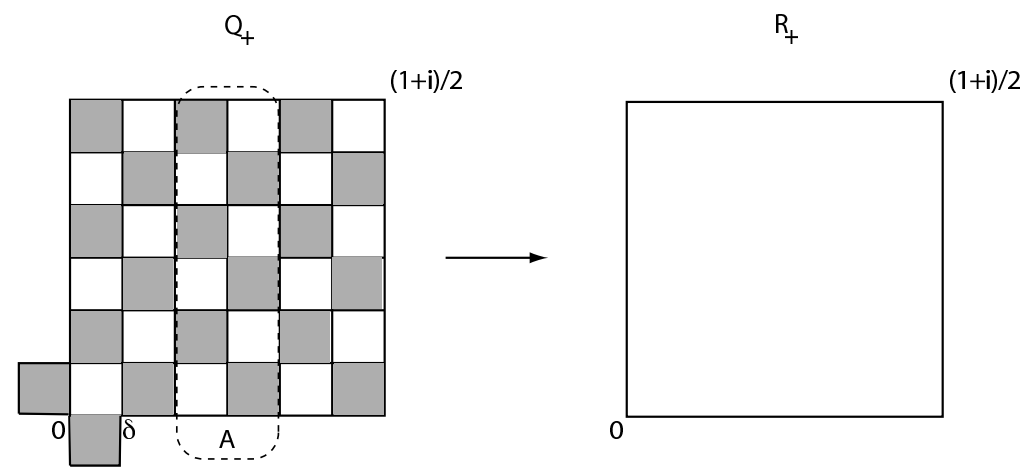

Figure 2. The map $g: X \rightarrow Y$ when $n=2$. The domain and codomain are respectively the doubles of the two polygons $Q_{+}, R_{+}$along their boundaries. Note that both $Q_{+}$ and $R_{+}$have an anticonformal symmetry given by reflection in the diagonal line $x=y$.

In this paragraph, we define $Y$. Let $R_{+}$denote the Euclidean square $[0,1 / 2] \times$ $[0,1 / 2]$ (we will call it "white") and $R_{-}$its mirror image $[0,1 / 2] \times[-1 / 2,0]$ under $j$ (we will call it "gray"). Let $Y$ be the Riemann surface obtained by taking the disjoint union of $R_{+}$and $R_{-}$and gluing the boundaries of the squares $R_{ \pm}$via $j$. Then $Y$ is isomorphic to the Riemann sphere; indeed, an isomorphism $\varphi$ is induced from the unique $\mathbb{Z}[i]$-periodic Weierstrass function $\wp$ sending the ordered quadruple $(0,1 / 2,(1+i) / 2, i / 2)$ to $(\infty,-1,0,1)$. The isomorphism $\varphi$ sends $R_{ \pm}$to $\mathbb{H}_{ \pm}$, the upper and lower half planes. See the right-hand side of Figure 2. The anticonformal involution $j: R_{ \pm} \rightarrow R_{\mp}$ induces an anticonformal involution $j_{Y}$ of $Y$ to itself; the isomorphism $\varphi$ conjugates $j_{Y}$ to $j$.

In this paragraph, we define $X$. Set $\delta=\frac{1}{2(n+4)}$, and let

$$
Q_{+}=[0,1 / 2]^{2} \cup([-\delta, 0] \times[0, \delta]) \cup([0, \delta] \times[-\delta, 0])
$$

and $Q_{-}=j\left(Q_{+}\right)$; see the left-hand side of Figure 2, which illustrates $Q_{+}$. Let $X$ be the sphere obtained from the disjoint union of $Q_{+}$and $Q_{-}$by gluing their boundaries via the map $j$. Then $X$ inherits a conformal structure from that of $Q_{ \pm}$: away 
from the corners this is clear; by the removable singularities theorem, this conformal structure extends over the corners. By the Uniformization Theorem, $X$ is isomorphic to the Riemann sphere. Again, there is an involution $j_{X}$ induced by $j$.

In this paragraph, we define the isomorphism $h: Y \rightarrow X$. Observe that both $R_{+}$and $Q_{+}$are Jordan domains in $\mathbb{C}$, on whose boundaries lie four distinguished points $(0,1 / 2,(1+i) / 2, i / 2)$, turning $R_{+}$and $Q_{+}$into quadrilaterals whose conformal shapes are characterized by their moduli. $R_{+}$is a square - the modulus is equal to 1 . The square is the unique quadrilateral admitting an anticonformal involution fixing a pair of opposite vertices. The reflection $x+i y \leftrightarrow y+x i$ gives such an involution of $Q_{+}$to itself. We conclude that there is a conformal isomorphism $h_{+}: R_{+} \rightarrow Q_{+}$fixing each element of the quadruple $(0,1 / 2,(1+i) / 2, i / 2)$. The Schwarz reflection principle implies $h_{+}$extends to an isomorphism $h: Y \rightarrow X$ sending the classes of the elements of the quadruple $(0,1 / 2,(1+i) / 2, i / 2)$ in $Y$ to those in $X$.

We now define the holomorphic map $g: X \rightarrow Y$. The quadrilateral $Q_{+}$is tiled by $(n+4)^{2}+2$ squares of side length $\delta$, as shown on the left of Figure 2. The dilation map $z \mapsto(n+4) z$ sends the small white square $[0, \delta] \times[0, \delta] \subset Q_{+} \subset X$ conformally onto the large white square $R_{+} \subset Y$. Applying the Schwarz reflection principle repeatedly, we conclude that this dilation extends to a degree $(n+4)^{2}+2$ holomorphic map $g: X \rightarrow Y$.

The remainder of the proof consists in verifying that the rational function $f_{n}: \widehat{\mathbb{C}} \rightarrow \widehat{\mathbb{C}}$ given by the composition

$$
\widehat{\mathbb{C}} \stackrel{\varphi^{-1}}{\longrightarrow} Y \stackrel{h}{\longrightarrow} X \stackrel{g}{\longrightarrow} Y \stackrel{\varphi}{\longrightarrow} \widehat{\mathbb{C}}
$$

has the desired properties.

The critical points of $F$ are points in $X$ which are corners of four or more tiles. It follows that under $f$, every critical point of $f$ is mapped into the set $\{-1,0,1, \infty\}$ and then to infinity, which is therefore a fixed critical point at which $f$ has local degree 3 . Hence $f$ is a critically finite hyperbolic rational map.

To find the desired annuli, set $A_{+}^{\prime}=[2 \delta, 1 / 2-2 \delta] \times[0,1 / 2] \subset Q_{+}$and $A_{-}^{\prime}=$ $j\left(A_{+}\right) \subset Q_{-}$; the union of $A_{ \pm}$defines an annulus $A^{\prime}$ in the quotient space $X$, so that $A:=h^{-1}\left(A^{\prime}\right)$ is an annulus in $Y$. By construction, the preimage $F^{-1}(A)$ consists of $(n+4)$ disjoint annuli parallel to $A$ mapping by degree $n+4$, together with one annulus lying in the double of the strip $[-\delta, 0] \times[0, \delta]$ mapping by degree 1 . Among the former, there are $n$ subannuli $A_{0}, \ldots, A_{n-1}$ compactly contained in $A$, each mapping under $F$ by degree $n+4$. Conjugating by $\varphi$, Corollary 2.1 applies, yielding $\operatorname{confdim}\left(J_{f_{n}}\right) \geq 1+\lambda_{n}$, where $J_{f_{n}}$ is the Julia set of $f_{n}$ and $\lambda_{n}$ is the unique positive root of the equation

$$
n(n+4)^{-\lambda}=1 .
$$

As $n \rightarrow \infty$, clearly $\lambda_{n} \rightarrow 1$ and so $\operatorname{confdim}\left(J_{f_{n}}\right) \rightarrow 2$.

Finally, we show that $J_{f}$ is a Sierpiński carpet. We imitate the arguments of Milnor and Tan given in the Appendix of [20]. They first show the following: 
Lemma 3.1. Let $f$ be a hyperbolic rational map and $z$ a fixed point at which the local degree of $f$ equals $k \geq 2$. Suppose $W$ is the immediate basin of attraction of $z$. Suppose there exist domains $U$ and $V$, each homeomorphic to the disk, such that $\bar{W} \subset U \subset \bar{U} \subset V$ and $\left.f\right|_{U}: U \rightarrow V$ is proper and of degree $k$. Then $\partial W$ is a Jordan curve.

Consider Figure 2. The conformal isomorphism $\varphi: Y \rightarrow \widehat{\mathbb{C}}$ sends the union of the top and right-hand edges of the right square to the interval $[-1,1]$ and sends the lower left corner point on the right labelled 0 to infinity. Let $V=\widehat{\mathbb{C}} \backslash[-1,1]$. The map $f$ has a unique periodic Fatou component $W$ - the immediate basin of $\infty-$ and clearly $W \subset V$. The domain $V$ is simply connected and contains exactly one critical value of $f$, namely the point $\infty$. It follows that there is exactly one component $U$ of $f^{-1}(V)$ containing $\infty$, and $\bar{W} \subset U \subset \bar{U} \subset V$ and $\left.f\right|_{U}: U \rightarrow V$ is proper and of degree 3. By Lemma 3.1, $\partial W$ is a Jordan curve. The remaining arguments needed are identical to those given in the Appendix of [20]: since $f$ is hyperbolic and critically finite, the Julia set is topologically one-dimensional, connected and locally connected, and there are no critical points in the Julia set. It follows that every Fatou component is a Jordan domain, and that the closures of the Fatou components are pairwise disjoint. Therefore $J_{f_{n}}$ is homeomorphic to the Sierpiński carpet [25].

\section{References}

[1] Bonk, M.: Quasiconformal geometry of fractals. In International Congress of Mathematicians. Vol. II, 1349-1373. Eur. Math. Soc., Zürich, 2006.

[2] Bonk, M.: Uniformization of Sierpiński carpets in the plane. Invent. Math. 186 (2011), no. 3, 559-665.

[3] Bonk, M., Kleiner, B., And Merenkov, S.: Rigidity of Schottky sets. Amer. J. Math. 131 (2009), no. 2, 409-443.

[4] Bourdon, M.: Immeubles hyperboliques, dimension conforme et rigidité de Mostow. Geom. Funct. Anal. 7 (1997), no. 2, 245-268.

[5] Bourdon, M. And PAJot, H.: Quasi-conformal geometry and hyperbolic geometry. In Rigidity in dynamics and geometry (Cambridge, 2000), 1-17. Springer, Berlin, 2002.

[6] Branner, B. And Fagella, N.: Quasiconformal surgery in holomorphic dynamics. To appear.

[7] Carrasco, M.: Jauge conforme des espaces métriques compacts. PhD thesis, AixMarseille Univ., 2012.

[8] David, G. And Semmes, S.: Fractured fractals and broken dreams. Oxford Lecture Series in Mathematics and its Applications 7, The Clarendon Press, Oxford University Press, New York, 1997.

[9] Falconer, K.: Techniques in fractal geometry. John Wiley, Chichester, 1997.

[10] Haïssinsky, P.: Géométrie quasiconforme, analyse au bord des espaces métriques hyperboliques et rigidités. In Astérisque 326 (2009), Exp. No. 993, ix, 321-362. Séminaire Bourbaki. Vol. 2007/2008. 
[11] Haïssinsky, P. And Pilgrim, K. M.: Examples of coarse expanding conformal maps. Discrete Contin. Dyn. Syst. Series A 32 (2012), no. 7, 2403-2416.

[12] Heinonen, J.: Lectures on analysis on metric spaces. Universitext. Springer-Verlag, New York, 2001.

[13] Hocking, J. G. And Young, G. S.: Topology. Dover Publications, New York, 1988.

[14] Kleiner, B.: The asymptotic geometry of negatively curved spaces: uniformization, geometrization and rigidity. In International Congress of Mathematicians. Vol. II, 743-768. Eur. Math. Soc., Zürich, 2006.

[15] Mackay, J. And Tyson, J.: Conformal dimension. Theory and application. University Lecture Series 54, American Mathematical Society, Providence, RI, 2010.

[16] McMullen, C. T.: Automorphisms of rational maps. In Holomorphic Functions and Moduli, Vol. I (Berkeley, CA, 1986), 31-60. Math. Sci. Res. Inst. Publ. 10, Springer, New York, 1988.

[17] McMullen, C. T.: The classification of conformal dynamical systems. In Current developments in mathematics, 1995 (Cambridge, MA), 323-360. Int. Press, Cambridge, MA, 1994.

[18] McMullen, C. T.: Self-similarity of Siegel disks and Hausdorff dimension of Julia sets. Acta Math. 180 (1998), no. 2, 247-292.

[19] McMullen, C. T. And Sullivan, D.: Quasiconformal homeomorphisms and dynamics. III. The Teichmüller space of a holomorphic dynamical system. Adv. Math. 135 (1998), no. 2, 351-395.

[20] Milnor, J.: Geometry and dynamics of quadratic rational maps. Experiment. Math. 2 (1993), no. 1, 37-83.

[21] Pansu, P.: Dimension conforme et sphère à l'infini des variétés à courbure négative. Ann. Acad. Sci. Fenn. Ser. A I Math. 14 (1989), no. 2, 177-212.

[22] Pilgrim, K. M. And TAN, L.: On disc-annulus surgery of rational maps. In Dynamical systems, Proceedings of the International Conference in honor of Professor Shantao Liao, 237-250. World Scientific, 1999.

[23] Sullivan, D.: Seminar on hyperbolic geometry and conformal dynamical systems. Preprint IHES, 1982.

[24] Sullivan, D.: Conformal dynamical systems. In Geometric dynamics (Rio de Janeiro, 1981), 725-752. Lecture Notes in Math. 1007, Springer, Berlin, 1983.

[25] Whyburn, G. T.: Topological characterization of the Sierpiński curve. Fund. Math. 45 (1958), 320-324.

Received November 23, 2010.

Peter Haïssinsky: Université Paul Sabatier, Institut de Mathématiques de Toulouse, 118 route de Narbonne, F-31062 Toulouse Cedex 9, France.

E-mail: phaissin@math.univ-toulouse.fr

Kevin M. Pilgrim: Department of Mathematics, Indiana University, Rawles Hall, Bloomington, IN 47401, USA.

E-mail: pilgrim@indiana.edu 